\title{
ANALYSIS OF LATITUDINAL DEPENDENCE OF SATURNIAN RADIO EMISSIONS
}

\author{
M. Y. Boudjada*, P. H. M. Galopeau ${ }^{\dagger}$, W. Macher*, \\ H. O. Rucker*, and W. Voller*
}

\begin{abstract}
Intense saturnian radio emission has been observed since June 2004, and until today, by the Radio and Plasma Waves Experiment (RPWS) on board Cassini spacecraft. During this long period of about six years the spacecraft was orbiting principally in the planetary equatorial plane. However in 2007 and 2008 orbits reach latitudes higher than $50^{\circ}$ which lead us to investigate sub-auroral saturnian radio emissions. In this study we examine the spectral distinctions between the saturnian radio emissions observed in the equatorial plane and those in latitudes close to the southern and northern auroral regions. We consider the three components reported by Galopeau et al. [2007, J. Geophys. Res., 112, A11213] principally dominated by the so-called saturnian kilometric radiation $(80 \mathrm{kHz}-900 \mathrm{kHz})$. We analyze the occurrence probability of these components taking into consideration different geometric configurations between the spacecraft, the planet and the Sun. We discuss the spectral alteration and change, from the equatorial plane to the high latitudes, of Saturnian radio emissions. Also we examine the spectral distinction between the radio emissions emitted from the northern and southern hemispheres, in particular in the case of the saturnian kilometric radiation (SKR). Our results are discussed and compared with those already reported in the literature dealing with the Voyager and the Cassini missions.
\end{abstract}

\footnotetext{
* Space Research Institute, Austrian Academy of Sciences, Schmiedlstrasse 6, A-8042 Graz, Austria † LATMOS, Université Versailles Saint-Quentin-en-Yvelines, CNRS, 78280 Guyancourt, France
} 
